\title{
PREVALENCIA DE PARÁSITOS INTESTINALES ZOONÓTICOS EN CANINOS (Canis lupus familiaris) DEL ÁREA URBANA DEL MUNICIPIO DE COYAIMA (TOLIMA)
}

\author{
ANDRÉS CAMILO GONZÁLEZ1 , JULIO CESAR GIRALDO
}

${ }^{1}$ Biólogo. Universidad INCCA de Colombia. Bogotá, Colombia

${ }^{2 *}$ Msc. Esp. Docente Investigador, líder Grupo de Investigación en Parasitología y Microbiología Tropical (GIPAMT). Facultad de Ingeniería, Administración y Ciencias Básicas. Programa de Biología. Universidad INCCA de Colombia. Bogotá, Colombia

${ }^{2 *}$ Correspondencia: Julio Cesar Giraldo. jcesargiraldo@gmail.com

Recibido: Mayo 4 de 2015 Aceptado: Julio 3 de 2015

\begin{abstract}
Resumen
Introducción: Las enfermedades parasitarias son un problema de salud pública al ser causadas por agentes patógenos que afectan animales domésticos, los que a su vez pueden infectar humanos, principalmente en países ubicados en áreas tropicales.

Objetivo: Identificar parásitos intestinales que afectan a caninos domiciliados de la zona urbana del municipio de Coyaima, y determinar la prevalencia y las variables asociadas al parasitismo en el área de estudio.

Materiales y Métodos: Se realizó un estudio descriptivo de corte transversal de febrero del 2011 a marzo del 2012, recolectando muestras de 175 caninos domiciliados de la zona urbana del municipio de Coyaima, las cuales se procesaron mediante la técnica de concentración formol - éter para la identificación de estadios de huevos de helmintos y quistes de protozoarios. Los datos fueron tabulados y analizados con el programa estadístico SPSS ${ }^{120}$.

Resultados: La prevalencia hallada para el parasitismo intestinal canino de la zona urbana del municipio de Coyaima fue del 53,1\%, siendo estos resultados concordantes con los reportados por otros autores en estudios similares, realizados en países latinoamericanos.

Conclusiones: La población canina de la zona urbana del municipio de Coyaima presenta afección por parásitos gastrointestinales, siendo entidades prevalentes Uncinarias, 20,6\%, Toxocara canis 8,6\%, Strongyloides spp., 2,9\%, Entamoeba spp., 21,1\%, Blastocystis spp., 18,3\% y Giardia spp., 16\%, entre las cuales se destacan, agentes etiológicos de relevancia por su carácter zoonótico, como los causantes de la toxocariosis, la migración larvaria cutánea y la giardiasis, entre otras. Se recomienda implementar campañas de educación en la población en general y ejecutar acciones de prevención por parte de estamentos responsables de la salud animal.
\end{abstract}

Palabras Claves: Uncinarias, Toxocara canis, Strongyloides spp., Giardia spp., prevalencia, zoonosis. 


\title{
PREVALENCE OF INTESTINAL PARASITES IN DOGS (Canis lupus familiaris) IN THE URBAN AREA OF DE COYAIMA TOWNSHIP (TOLIMA)
}

\begin{abstract}
Introduction: The parasitic diseases are public health problem, because are caused by pathogens that affected domestic animals and right through then can infected the human, mainly in tropical countries.

Objective: Identify the intestinal parasites in dogs of the Coyaima urban area, also the prevalence and associated variables that are involved with the parasitism in the study area.

Materials and Methods: it was developed study between February 2011 to March 2012 where were collected 175 dog's samples in the Coyaima urban area. The samples were processed between the formol - eter technique for the qualitative analysis of the helminths eggs and protozoan cysts. The data were tabulate and analyzed through the statistic program SPSS ${ }^{\mathrm{v20}}$.

Results: It was found prevalence for intestinal parasites in dogs in the Coyaima urban area was 53, $1 \%$. This results match with other studies in the same department.

Conclusions: The canine population in the Coyaima urban area is highly affected for intestinal parasites; the entities more prevalent are Uncinarias, 20, 6\%, Toxocara canis 8, 6\%, Strongyloides spp., 2, 9\%, Entamoeba spp., 21, 1\%, Blastocystis spp., 18, 3\% and Giardia spp., 16\%. It is recommended implement educational campaigns in all population, and executed preventive actions by the responsible statements concerning animal health.
\end{abstract}

Keywords: Uncinarias, Toxocara canis, Strongyloidesspp., Giardiaspp, prevalence, zoonoses.

\section{PREVALÊNCIA DE PARASITOS INTESTINAIS EM CÃES (Canis lupus familiaris) NA ÁREA URBANA DO MUNICÍPIO DE COYAIMA (TOLIMA)}

\section{Resumo}

Introdução: As doenças parasitárias são um problema de saúde pública, as quais são causadas por agentes patogênicos que afetam animais domésticos e que a sua vez podem infetar ao ser humano principalmente em países localizados em áreas tropicais.

Objetivo: Identificar parasitos intestinais que afetam aos cães residentes na área urbana do município de Coyaima e determinar a prevalência e as variáveis associadas ao parasitismo na área de estudo.

Materiais e métodos: Um estudo descritivo do tipo transversal foi realizado entre fevereiro de 2011 e março de 2012, no estudo foram recolhidas amostras de 175 cães residentes na área urbana de município de Coyaima, as quais foram processadas mediante uma técnica de concentração conhecida como formol - éter, a fim de analisar quantitativamente a quantidade de ovos de helmintos e cistos de protozoários. Os dados foram tabulados e analisados com o software estatístico SPSS ${ }^{\mathrm{v2} 2}$.

Resultados: A prevalência encontrada para o parasitismo intestinal canino da área urbana do município de Coyaima foi de 53,1\%, estes resultados são consistentes com aqueles reportados por outros autores em estudos semelhantes realizados em países da América Latina.

Conclusões: A população canina da área urbana do município de Coyaima tem afeição por parasitos gastrintestinais. As entidades mais prevalentes são Uncinarias, 20, 6\%, Toxocara canis 8, 6\%, Strongyloides spp., 2, 9\%, Entamoeba spp., 21, 1\%, Blastocystis spp., 18, 3\% e Giardia spp., 16\%, entre as quais se destacam agentes etiológicos de relevância por seu caráter zoonótico, como causadores de toxocaríase, a larva migrans cutânea e a giardíase, entre outras.

Palavras-chave: Uncinarias, Toxocara canis, Strongyloides spp., Giardia spp, prevalência, zoonose. 


\section{Introducción}

Las Enteroparasitosis son infecciones causadas por protozoos y helmintos, que afectan especialmente el aparato digestivo del huésped, donde se multiplican y se adaptan al organismo; las vías de adquisición pueden ser oral, dermal, y algunas veces transplacentaria o mamarias; los mecanismos de trasmisión tienen relación con sus respectivos ciclos biológicos que a su vez se ven favorecidos por el entorno $(1,2)$.

Estas parasitosis son comunes en países tropicales y subtropicales, donde las condiciones socioeconómicas, demográficas y ambientales las propician. Factores como la inadecuada manipulación y cocción de alimentos, inapropiados hábitos y condiciones higiénico-sanitarias, carencia de atención en salud y bajo nivel de escolaridad, aumentan la probabilidad de trasmisión (3).

Entre los agentes infecciosos que parasitan el tracto gastrointestinal de caninos se hallan helmintos y protozoos que son potencialmente patógenos tanto para ellos como para el hombre por su carácter zoonótico como lo es la toxocariosis, la migración larvaria cutánea, la estrongiloidiasis y giardiasis, siendo la población infantil la de mayor riesgo de contraer estas infecciones $(4,5)$.

Algunos de los síntomas que experimentan los caninos contagiados son anorexia, pérdida de sangre y proteínas plasmáticas a través del tracto intestinal, alteraciones en el metabolismo proteico, reducción de minerales, disminución de la actividad enzimática digestiva, diarrea y en adultos excreción de parásitos en el vómito y en heces; en el caso de infecciones masivas se presentan signos como abdomen abultado, mala condición del pelaje, diarrea y retardo en el desarrollo (6).

Las helmintiasis en los caninos son relevantes por las consecuencias que pueden generar en éste, es el caso de $T$. canis, cuya principal manifestación es la anorexia, además cuadros de infección masiva puede ocasionar la muerte de los cachorros (7).

Los caninos cuya función primordial es la de ser animales de compañía, cumplen un papel preponderante en la transmisión de infecciones causadas por protozoos y helmintos, entre las cuales se destacan la hidatidosis, la migración larvaria visceral, ocular y larva migrans cutánea ocasionada por los estados larvales de Echinococus granulosus, T. canis y Uncinarias respectivamente (7), y cuadros diarreicos agudos por el protozoo Giardia duodenalis, entre otros. Los habitantes de la región estudiada, deben estar informados sobre el ciclo de vida de los parásitos, la etiología de este con el hospedero; de igual manera se debe hacer énfasis en los factores que facilitan el contagio, entre los cuales se deben destacar el incremento de la población de mascotas y el contacto directo de estas con las personas, especialmente con los niños, así mismo la carga parasitaria propia de cada región y la diversidad de esta (8).

Este estudio se realizó, con el objetivo de aportar al conocimiento sobre las prevalencias de parásitos gastrointestinales en los caninos domiciliados del municipio de Coyaima, departamento del Tolima, e identificar las posibles variables socioeconómicas, demográficas y ambientales asociadas a estas, el producto de la investigación debe manifestarse socialmente en educación y cambio de actitud en la comunidad para el establecimiento de medidas de prevención, tratamiento y control para población canina del sector urbano.

\section{Metodología}

\section{Áreay población de estudio}

Se realizó un estudio descriptivo de corte transversal, con toma única de muestra en la población canina del área urbana del municipio de Coyaima, departamento del Tolima; este cuenta con un tamaño territorial de $664.33 \mathrm{~km}^{2}$ equivalente al 2,82\% del área departamental, de los cuales $658.13 \mathrm{~km}^{2}$ corresponden al sector rural (99\%) y $6,20 \mathrm{~km}^{2}$ al sector urbano (1\%), se encuentra a 392MSNM, ubicándose en el piso térmico cálido con una temperatura promedio de $27^{\circ} \mathrm{C}$. Al momento de la realización del estudio, no se disponía de información producto de un censo que determinara la población canina residente en el casco urbano.

En lo relacionado con los servicios de salud animal, el municipio cuenta con dos clínicas veterinarias, las cuales una vez indagadas manifestaron su desconocimiento sobre las principales afecciones parasitarias de la población canina del municipio y las posibles variables asociadas.

Población objeto de estudio: todos los caninos (Canis lupus familiaris) del área urbana del Municipio de Coyaima (Tolima). La muestra obtenida se conformó con los ejemplares que cumplieron previa autorización y aceptación por parte del propietario para participar voluntariamente en el estudio.

\section{Recolección de la información}

La información se colectó en el segundo semestre del año 2011. El muestreo se realizó por conveniencia y se invitó a 
participar a personas propietarias de caninos cuya finalidad fuese la de ser mascota. Se convocó a la población y se impartieron charlas acerca del parasitismo intestinal en animales de compañía: características generales, agentes parasitarios de mayor frecuencia, vías de trasmisión y consecuencias en la salud del animal. Posteriormente se informó sobre el desarrollo del estudio, se realizó visita domiciliaria a los propietarios y previo consentimiento, se diligenció una encuesta epidemiológica.

La encuesta incluyó en primera instancia información personal del cuidador y aspectos de nivel socioeconómico del propietario; la segunda etapa se orientó en la información de los caninos, de la que se destaca: nombre del animal, raza, edad, procedencia, uso de tratamiento antiparasitario, producto, manifestación de expulsión de parásitos, tipo de alimentación suministrada, disposición de las excretas, síntomas, condición física, personas y animales con quiénes convive, esquema de vacunación y finalidad del animal.

\section{Toma de muestra}

Finalizada la encuesta, se les informó a los participantes sobre el procedimiento para la toma de muestra en los caninos, la que se colectó de forma directa mediante tacto rectal. La visita domiciliaria se realizó casa por casa, permitió la cobertura de todo el casco urbano del municipio, lográndose la recolección de 175 muestras, las que se rotularon y conservaron con $30 \mathrm{ml}$ de reactivo MIF (merthiolate, yodo y formalina) al $5 \%$ y posteriormente se refrigeraron a $4^{\circ} \mathrm{C}$ y transportaron al laboratorio de parasitología de la Universidad Incca de Colombia para su procesamiento y diagnóstico parasitológico, con el empleo de la técnica de concentración formol - éter, para la identificación de quistes de protozoos, huevos y estadios larvales de helmintos (9), siendo ésta la técnica de referencia; por aumentar la posibilidad de concentrar la presencia de formas parasitarias, constituyéndose en una ventaja con respecto al examen directo, si solo se ha tomado una muestra. El análisis microscópico se evaluó por triplicado y doble ciego (10). Los resultados obtenidos fueron informados a los propietarios o cuidadores para su respectivo tratamiento.

\section{Análisis Estadístico}

El tratamiento estadístico se realizó con el empleo del programa SPSS ${ }^{\mathrm{v} 20}$, el que incluyó estudio de frecuencias y el análisis bivariado con distribución ji cuadrado para establecer variables estadísticamente significativas y cálculo de Odds radios (OR) para cada una de éstas, con un índice de confianza (IC) del 95\%.

\section{Resultados}

Se analizaron 175 muestras de materia fecal de caninos provenientes de la zona urbana del municipio de Coyaima departamento del Tolima, de las cuales el 53,1\% (93/175) fueron positivas por presentar al menos una entidad parasitaria; en tanto que el 46,9\% (82/175) restante, no se observó evidencia de algún agente etiológico.

\section{Prevalencia de parásitos intestinales}

Los resultados arrojaron que el 53,1\% (93/175) de los ejemplares presentan diferentes formas parasitarias distribuyéndose estos de la siguiente manera: para helmintos Uncinarias $20,6 \% ; T$. canis $8,6 \%$ y Strongyloides spp., con 2,9\%; en tanto que los protozoos Entamoebaspp., 21,1\%; seguida por Blastocystisspp., y Giardia spp., con un18, 3\% y 16\% respectivamente.

En la tabla 1 se presentan los valores porcentuales, correspondientes a las entidades parasitarias de mayor prevalencia en los caninos incluidos en el estudio.

Tabla 1. Prevalencias de parásitos gastrointestinales en Canis lupus familiaris en la zona urbana del municipio de Coyaima (Tolima) 2013.

\begin{tabular}{lcc} 
ENTIDAD PARASITARIA & FRECUENCIA & PREVALENCIA \\
HELMINTOS & 36 & \\
Uncinarias & 15 & $20,6 \%$ \\
Toxocara canis & 5 & $8,6 \%$ \\
Toxascaris leonina & 5 & $2,9 \%$ \\
Strongyloides spp. & 2 & $2,9 \%$ \\
Dipylidium caninum & 2 & $1,1 \%$ \\
Trichuris vulpis & & $1,1 \%$ \\
PROTOZOOS & 37 & \\
Entamoeba spp. & 32 & $21,1 \%$ \\
Blastocystis spp. & 28 & $18,3 \%$ \\
Giardia spp. & 1 & $16 \%$ \\
Chilomastixspp. & & $0,6 \%$ \\
\hline
\end{tabular}

\section{Aspectos epidemiológicos}

El análisis de los datos consignados en las fichas epidemiológicas permitió identificar que el 84,6\% corresponde a animales denominados de raza criolla o mestiza, el $15,4 \%$ a razas puras entre las cuales se destacaron la Labrador, Pitbull, French Poodle y Pincher entre otras.

Los caninos se categorizaron en dos grupos etarios, el primero lo conformaron animales de cero a dos años $69,2 \%$ 
y mayores a dos 30,8\%. Se halló asociación estadísticamente significativa entre la edad y la presencia de agentes parasitarios como Strongyloides spp., y $D$. caninum ( $\mathrm{p}=0,016$ y $\mathrm{p}=0,033$ respectivamente).

De acuerdo a la clasificación por género, el porcentaje de machos fue del 59,4\% y hembras 40,6\%. Aunque el valor porcentual de prevalencia se presentó en mayor proporción en machos, esta diferencia no fue estadísticamente significativa $(p=0,244)$, sin embargo, sobresale la asociación con Toxocara canis por su cercanía a la significancia estadística ( $p=0,074)$. La condición corporal observada en los ejemplares en su mayoría fue buena (156 ejemplares), solo $19 / 175(10,8 \%)$ de los animales presentaron una condición corporal famélica.

\section{Hábitos alimenticios}

Se halló que el 37,5\% de los propietarios manifestaron alimentarlos con concentrado, el restante 62,5\% disponían de alimentación doméstica. Con relación al suministro de agua el $100 \%$ de la población les proporciona agua cruda, no encontrándose una relación significativa entre esta variable y el parasitismo en general, a excepción de Giardia spp., en la que su presencia se asocia con esta variable $(p=0,002)$.

\section{Lugar de eliminación de excretas}

Al momento de la recolección de la información, se observó para la mayoría de animales del municipio, la realización de fecalismo al aire libre representado en el 68,5\%; solo el $31,5 \%$ de los caninos contaban con un adecuado manejo de excretas por parte de su cuidador; a pesar de ello, no hay una asociación significativa entre esta variable y la presencia de parásitos intestinales, pero sí se pueden evidenciar las condiciones de higiene que manejan los cuidadores con sus mascotas. Es importante resaltar que el manejo inadecuado de esta condición puede convertirse en un factor de riesgo para las personas, por la presencia de parásitos de carácter zoonótico en las heces de los perros, incrementando la probabilidad de infección humana.

El análisis de los datos consignados en las fichas epidemiológicas permitió identificar que el 84,6\% corresponde a animales denominados de raza criolla o mestiza, el $15,4 \%$ a razas puras entre las cuales se destacaron la Labrador, Pitbull, French Poodle y Pincher entre otras.

Los caninos se categorizaron en dos grupos etarios, el primero lo conformaron animales de cero a dos años $69,2 \%$ y mayores a dos 30,8\%. Se halló asociación estadísticamente significativa entre la edad y la presencia de agentes parasitarios como Strongyloides spp., y $D$. caninum ( $\mathrm{p}=0,016$ y $\mathrm{p}=0,033$ respectivamente).

De acuerdo a la clasificación por género, el porcentaje de machos fue del 59,4\% y hembras 40,6\%. Aunque el valor porcentual de prevalencia se presentó en mayor proporción en machos, esta diferencia no fue estadísticamente significativa $(p=0,244)$, sin embargo, sobresale la asociación con $T$. canis por su cercanía a la significancia estadística $(p=0,074)$. La condición corporal observada en los ejemplares en su mayoría fue buena (156 ejemplares), solo $19 / 175(10,8 \%)$ de los animales presentaron una condición corporal famélica.

\section{Hábitos alimenticios}

Se halló que el 37,5\% de los propietarios manifestaron alimentarlos con concentrado, el restante 62,5\% disponían de alimentación doméstica. Con relación al suministro de agua el $100 \%$ de la población les proporciona agua cruda, no encontrándose una relación significativa entre esta variable y el parasitismo en general, a excepción de Giardia spp., en la que su presencia se asocia con esta variable $(p=0,002)$.

\section{Lugar de eliminación de excretas}

Al momento de la recolección de la información, se observó para la mayoría de animales del municipio, la realización de fecalismo al aire libre representado en el 68,5\%; solo el $31,5 \%$ de los caninos contaban con un adecuado manejo de excretas por parte de su cuidador; a pesar de ello, no hay una asociación significativa entre esta variable y la presencia de parásitos intestinales, pero sí se pueden evidenciar las condiciones de higiene que manejan los cuidadores con sus mascotas. Es importante resaltar que el manejo inadecuado de esta condición puede convertirse en un factor de riesgo para las personas, por la presencia de parásitos de carácter zoonótico en las heces de los perros, incrementando la probabilidad de infección humana.

\section{Tratamiento antiparasitario}

La información consignada en los formatos epidemiológicos, demuestra que solo el 42,9\% (75/175) de los caninos contaron con un adecuado tratamiento antiparasitario, el restante $57,1 \%$ no se les suministra ningún producto o se realizó con poca frecuencia (una vez cada dos años), lo cual puede favorecer la infección por entidades parasitarias y en especial por protozoos como Blastocystis spp., $(\mathrm{p}=0.037)$ variable con significancia estadística para este agente etiológico. 


\section{Presencia de síntomas}

Solo el 13,1\% (23/175) manifestó al menos un síntoma como diarrea o vómito. De acuerdo al análisis estadístico la variable síntoma, se relacionó con la presencia de Uncinarias ( $p=0,039)$; no siendo así el caso para Toxascaris leonina con la que no se halló una asociación estadísticamente significativa, pero sí es de resaltar la proximidad el valor de $\mathrm{p}(\mathrm{p}=0,074)$ calculado para ésta.

En la tabla 2 se presentan los valores de las variables con significancia estadística, asociadas a la infección por parásitos gastrointestinales en caninos del municipio de Coyaima (Tolima).

Tabla 2. Variables asociadas a la infección por parásitos gastrointestinales en Canis lupus familiaris del municipio de Coyaima (Tolima) 2013.

\begin{tabular}{|c|c|c|c|c|c|}
\hline FACTOR & ASOCIACIÓN & P-CALCULADO & OR & IC & $95 \%$ \\
\hline Estrato & Parasitismo General & 0,035 & & & \\
\hline \multirow[t]{2}{*}{ Alimentación } & Giardia spp. & 0,015 & & & \\
\hline & Entamoeba spp. & 0,003 & & & \\
\hline \multirow[t]{2}{*}{ Antiparasitario } & Giardia spp. & 0,002 & & 0,240 & $0,092-0,624$ \\
\hline & Blastocystis spp. & 0,037 & & 2,271 & $1,040-4,960$ \\
\hline \multirow[t]{2}{*}{ Edad } & Strongyloides spp. & 0,016 & 0,104 & & $0,011-0,955$ \\
\hline & Dipylidium caninum & 0,033 & 0,963 & & 0,914-1,015 \\
\hline \multirow[t]{2}{*}{ Síntomas } & Uncinarias & 0,039 & 0,152 & & $0,020-1,168$ \\
\hline & Toxocaris leonina & 0,074 & & 4,730 & $0,746-29.981$ \\
\hline Genero & Toxocara canis & 0,074 & 3,111 & & $0,845-11,451$ \\
\hline
\end{tabular}

En la tabla 3 se presentan los valores de las variables con las cuales no se halló asociación estadísticamente significativa con la infección por parásitos gastrointestinales en caninos del municipio de Coyaima (Tolima), pero brindan información acerca de la convivencia, condición socioeconómica y hábitos higiénico-sanitarios, entre otros, que tienen los cuidadores para con sus mascotas.
Tabla 3. Variables asociadas a la infección por parásitos gastrointestinales en Canis lupus familiaris del municipio de Coyaima (Tolima) 2013.

\section{FACTOR} PREVALENCIA

Tiempo de permanencia en el hogar

Menor o igual a 24 meses

$77,7 \%$

Entre 24 y 48 meses

$12 \%$

Mayor a 48 meses

$10,3 \%$

Tamaño de los caninos

Pequeños

$33,7 \%$

Mediano

$46,9 \%$

Grande

$19,4 \%$

Número de personas

Una sola persona

Más de una persona

$98,3 \%$

Vacunas

Vacunas completas

Sin vacunas o incompletas

Presencia de lesiones en la piel

Presentaban lesiones

No presentaban lesiones

$88,6 \%$

Estado y/o condición de la piel

Buena

Regular

Mala

Finalidad del canino en la familia

Compañía

Seguridad y otros

Convivencia

Conviven solo con un canino

Estrato del Propietario o cuidador

Alto (1)

Medio (2) 
Las gráficas 1 y 2 detallan los resultados sobre el parasitismo gastrointestinal de los caninos en el municipio. En la gráfica 3 se observan las entidades parasitarias que presentaron los valores porcentuales prevalentes de mayor relevancia.

Prevalencia de helmintos parásitos en el casco urbano del municipio de Coyaima - Tolima

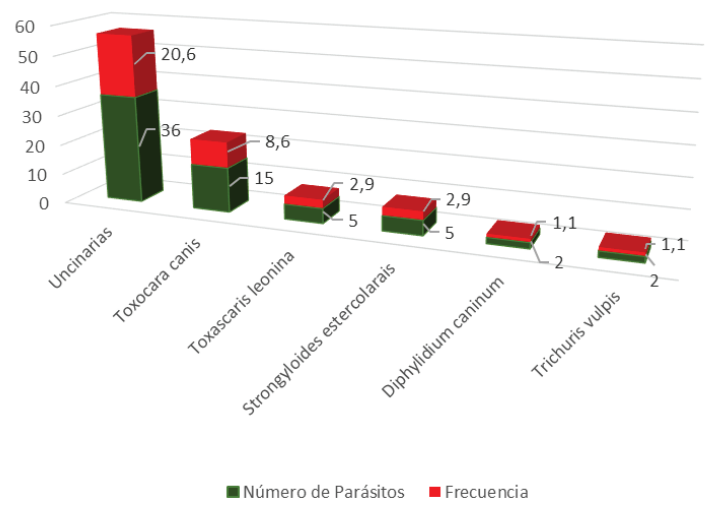

Grafica 1. Prevalencia de helmintos parásitos en caninos domiciliados en el municipio de Coyaima-Tolima.

Prevalencia de protozoos parásitos en el casco urbano del municipio de Coyaima - Tolima

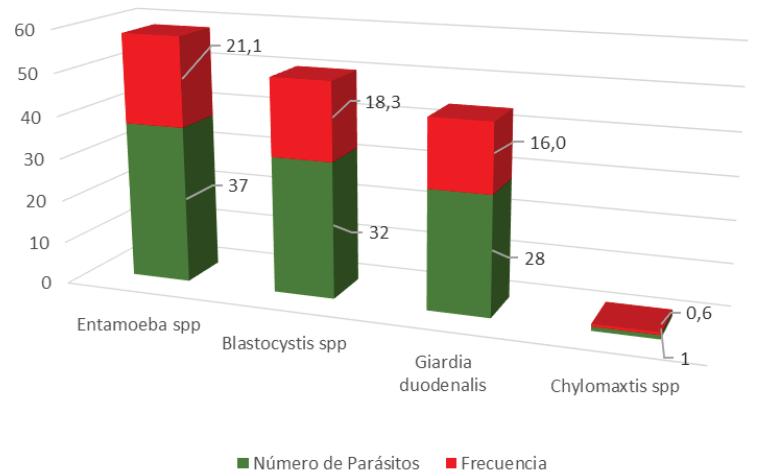

Grafica 2. Prevalencia de protozoos parásitos en caninos domiciliados en el municipio de Coyaima-Tolima.

Frecuencia de parásitos intestinales en el casco urbano del municipio de Coyaima - Tolima

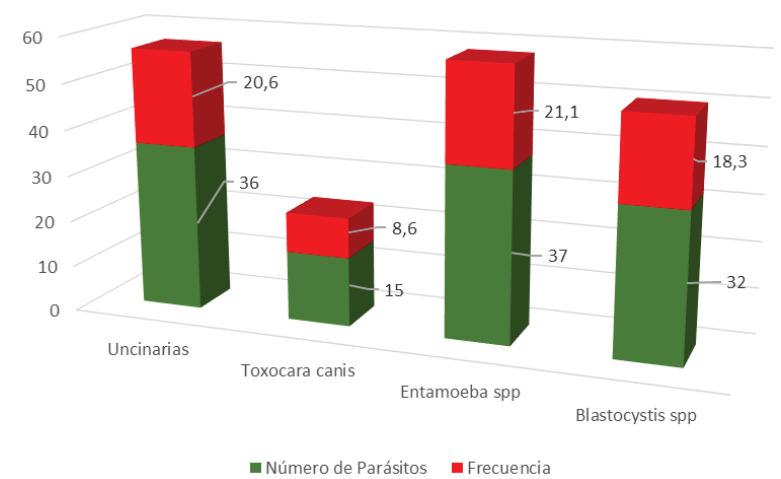

Grafica 3. Entidades parasitarias en caninos domiciliados del casco urbano en el Municipio de Coyaima, que presentaron los mayores valores porcentuales.

\section{Discusión}

La prevalencia de parásitos intestinales en el casco urbano del municipio de Coyaima presentó un valor de 53,1\%, concordando con lo reportado por otros autores a nivel mundial (11-13). Los registros para Colombia oscilan en valores porcentuales de prevalencias entre $37,4 \%$ y el $76 \%$ para diferentes formas parasitarias como larvas, huevos o quistes identificados en heces de los caninos examinados $(14,15)$. Es de aclarar que no existen reportes previos de parasitismo gastrointestinal canino para el departamento del Tolima, ni para este municipio, de acuerdo a la información suministrada por la Secretaría de Salud en la dependencia de Zoonósis, como tampoco se tiene registros sobre la afección y la problemática que el parasitismo canino pueda ocasionar en la salud pública en la comunidad.

Al comparar la prevalencia hallada en este estudio, con las reportadas por otros investigadores a nivel nacional, llevadas a cabo en ciudades capitales como Popayán (Cauca), los resultados arrojaron en éste, que el 37,9\% de las muestras presentaban alguna forma parasitaria (16); cabe también resaltar los valores obtenidos en un estudio realizado en el Centro de Zoonósis de la ciudad de Bogotá D.C, donde halló prevalencias del 76\% (14), y un reporte aún más reciente para dicho centro en el que se informa de una prevalencia del 88,6\%,en 70 muestras analizadas, donde $62 / 70$ se diagnosticaron positivas para algún agente parasitario (17). A nivel departamental se debe destacar los estudios que reportaron una prevalencia del $37,4 \%$ para parásitos intestinales caninos en el departamento del Huila (15), y por su parte se encontró una frecuencia del 22,2\% para el departamento del Quindío (6). Las diferencias observadas en los valores porcentuales de las prevalencias en los diferentes estudios analizados, pueden obedecer a variaciones ambientales atribuibles a temperatura y humedad, dado que estos se han realizado tanto en zonas secas como húmedas y además con diferencias en altitud y temperatura, otra variable que puede estar directamente relacionada son las condiciones higiénico-sanitarias de cada ciudad o departamento, donde se verá más favorecida la infección por agentes parasitarios en zonas que no cuenten con las condiciones básicas sanitarias satisfechas y en especial con un sistema de acueducto y manejo de aguas residuales adecuado (18).

En el presente estudio, los valores porcentuales para parasitosis prevalentes fueron: Uncinarias, 20,6\%,T canis $8,6 \%$ y Strongyloides spp., 2,9\% por geohelmintiasis y protozoos por Entamoeba spp., 21,1\%, Blastocystisspp., $18,3 \%$ y Giardia spp., 16\% siendo los de mayor porcentaje. En 2008 reportaron resultados similares en Venezuela donde Uncinarias, $(45,9 \%)$, y $T$. canis $(31,8 \%)$ presentaron 
los porcentajes más elevados (19). Por su parte T. canis arrojo la mayor prevalencia en la zona urbana de la ciudad de ICA, Perú, seguido por Ancylostoma caninum.

Blastocystis spp., es un protozoo gastrointestinal que no se había registrado en los estudios en Colombia, solo en la ciudad de Popayán, se reportó datos para este agente parasitario (16). Esta entidad no es considerada patógena por algunos autores, sin embargo para otros, es un microorganismo que merece mayor atención, por lo tanto sería importante abordar estudios a nivel nacional, para determinar su posible carácter patógeno (16).

La presencia de Giardia spp., se confronta con otros estudios como los realizados en el departamento del Huila y la ciudad de Medellín, de igual manera los reportes mundiales denotan el incremento de los valores porcentuales de prevalencia para este agente parasitario, que no dista de la hallada en el presente estudio, siendo cercana a lo reportado para Medellín, Colombia (24\%) y Puno, Perú (14.3\%).

Otras entidades que cuya incidencia fue baja pero se resalta su presencia fueron T. vulpis y Strongyloides spp., en 2011 reportan una prevalencia de 0,37\% para Strongyloides spp., en la ciudad de México (20), por su parte en un estudio realizado en Argentina obtuvo prevalencias cercanas para estas parasitosis, a las informadas para Coyaima de T. vulpis y Strongyloides spp., con $1,5 \%$ y $2,9 \%$ respectivamente (21).

En la investigación realizada en Coyaima (Tolima) la población canina evaluada estuvo representada por animales de toda la zona urbana del municipio, conformada por siete barrios, las mayores prevalencias se hallaron en: La Vega (69,5\%), Benigno Capera (57,1\%), La Esperanza y Centro (50\%), los demás arrojaron valores inferiores. En cuanto al análisis estadístico, se identificó una asociación significativa entre la variable lugar de residencia y la presencia de parásitos gastrointestinales $(p=0,035)$, esto puede estar relacionado con las condiciones higiénicosanitarias que presentan estos lugares que son deficientes y además por ser barrios con un nivel socioeconómico bajo y con condiciones básicas insatisfechas, lo cual favorece la infección por agentes parasitarios (18).

Asimismo estos valores concuerdan con los reportes para Popayán en el 2004, donde los caninos más afectados parasitariamente fueron los pertenecientes a personas de niveles socioeconómicos de escasos recursos (16). Se resalta también la prevalencia de Giardia spp., (16\%) con un valor de $\mathrm{p}=0,015$ donde hay una relación estadísticamente significativa entre esta variable y la frecuencia de este protozoo; de la misma manera la infección en los perros por este agente, está asociado con el estado higiénico sanitario del ambiente (22). Estos resultados también son valores cercanos a los reportados para distritos del Cono Sur de Lima Metropolitana del 15,7\% (23). En Bélgica un estudio similar, determinó que el $18.1 \%$ de los animales analizados presentaban este agente parasitario (24), de igual manera se halló una prevalencia del 7,4\% en caninos sin domicilio de Irán (25). En Colombia se reportó un valor del 4,5\% para la ciudad Popayán (16). Estos resultados demuestra la distribución cosmopolita de este agente parasitario afectando tanto a países en desarrollo como industrializados. En lo referente a Entamoeba spp., también se halló una relación estadísticamente significativa entre este agente parasitario y variable condición higiénico-sanitaria del ambiente $(p=0,003)$.

En los caninos evaluados en el municipio de Coyaima (Tolima), la distribución por rango de edades fue muy amplio, para poder calcular asociaciones parasitarias y riesgo, la variable edad, se dicotomizó distribuyéndose en dos grupos: animales mayores a dos años y menores a este rango. La frecuencia más elevada fue para caninos menores a dos años (121/175) equivalente al 69,1\% y en especial en los menores a seis meses $(55,4 \%)$, no hallándose diferencias estadísticas significativas en esta categoría. Sin embargo, una posible causa es que la inmunidad en éstos, empieza a manifestarse a partir de la quinta semana de edad, por ende, ejemplares menores a dos años en los cuales se incluyeron animales cachorros (menores a seis meses de edad), pueden estar más expuestos a la infección por entidades como $T$. canis y Uncinarias, (la toxocariosis) cuya trasmisión parasitaria puede ocurrir de forma transplacentaria o transmamaria, razón por la cual los cachorros pueden estar parasitados incluso antes de nacer, o desde el momento en que empiezan a alimentarse (16). En el estudio para el departamento del Quindío, 2005, reportan resultados similares donde los animales más afectados correspondieron a aquellos menores a un año de edad y las parasitosis de mayor relevancia fueron helmintos, al igual que en este estudio, otro aspecto a tener en cuenta es que se halló una asociación estadísticamente significativa entre la variable edad y la presencia de $D$. caninum ( $\mathrm{p}=0,033$; OR=0,963; IC 95\% 0,914-1,015) y de Strongyloides stercoralis $(\mathrm{p}=0,016$; OR $=0,016$; IC $95 \%=0,011-0,955)(6)$, siendo esto también coincidente con lo reportado en un estudio realizado en la Ciudad de Medellín, donde los mayores valores porcentuales para las helmintiasis se presentaron en animales menores a dos años de edad (26).

Con respecto a la distribución de parásitos intestinales por género y por raza, los resultados en esta región son muy similares a los reportados en investigaciones nacionales, 
destacándose el dominio de la raza denominada criolla o mestiza. A pesar de no encontrase asociaciones ni diferencias estadísticamente significativas, se resalta la relación entre el género y la presencia de $T$. canis $(p=0,074$; $\mathrm{OR}=3,111$; IC 95\%=0,845-11,451), por su cercanía a la significancia estadística. Los machos se presentaron más afectados que las hembras $56,9 \%$, siendo esto concordante con lo reportado en el 2007, 27,8\% en machos en comparación con las hembras del 21,3\% (25), e igualmente en el estudio realizado en Río de Janeiro (Brasil) los primeros presentaron prevalencias del 44,2\% (26). Es de destacar que para todos estos reportes no hubo una diferencia o asociación estadísticamente significativa.

Otros factores en los cuales se identificaron asociaciones con significancia fueron tipo de alimentación y presentar sintomatología. La presencia de Giardia spp., está relacionada por el tipo de alimentación $(p=0,002$; $\mathrm{OR}=0,240 ; \mathrm{IC} 95 \%=0,092-0,624)$, es de esperar que ésta estuviese en concordancia con el alimento doméstico, pero los resultados del estudio demostraron una mayor prevalencia en animales cuyos propietarios manifestaron estar alimentándolos con concentrado (37,5\%), lo que puede ser un indicador que el almacenamiento de este tipo de alimento no es el adecuado y por tal razón no cumple las condiciones higiénico sanitarias y por ende se convierte en un factor de riesgo por la presencia de formas resistentes como quistes de protozoos y huevos de helmintos $(16,21)$, o tal vez el concentrado no sea la dieta principal de los caninos que dicen estar alimentados así, pudiéndose presentar, que esta sea complementada con sobras domésticas, por ende estarían consumiendo dos tipos de alimento. Es importante resaltar las fuentes de agua para consumo de los animales, porque este podría ser un factor de riesgo para la infección parasitaria. Varios estudios reportan la presencia de Giardia spp., en Perú en el 2003 y Medellín en 2007, ambos casos referencian prevalencias moderadas destacando el no haberse presentado asociación entre la variable tipo de alimentación y la presencia del parasito (25). Respecto a la manifestación de síntomas, se tuvo en cuenta aquellos caninos que en el momento de la toma de la muestra presentaron diarrea o vómito, siendo el 13,7\% de los animales estudiados, cuyos propietarios informaron haberse dado al menos una de estas condiciones dos o tres días al procedimiento, hallándose asociación con la presencia de Uncinarias, por su valor estadístico significativo ( $\mathrm{p}=0,039$; OR=0,152; IC $95 \%=0,020-1,168)$.

Otras variables que se tuvo en cuenta por estar relacionadas son: la convivencia de los caninos y el cuidado básico de ellos por parte de su propietario; en estos dos casos, los resultados arrojaron que el $84 \%$ de las mascotas su finalidad era ser animales de compañía, así como el 40\% de los mismos convivían con más de una mascota, incrementándose el riesgo de infección en las personas con las que comparte, en especial por agentes zoonóticos como Uncinarias, $T$. canis y Giardia spp., $(4,5)$. Se observó buenas condiciones en los animales evaluados, sólo el 1,1\% presentaron una condición clase cuatro correspondiente a famélica y el 11,4\% presentó algún tipo de lesión, lo que hace suponer un buen cuidado de los animales por parte de su propietario, el 59\% de los ejemplares certificaron tener su esquema de vacunación completo, contrastando con el tratamiento antiparasitario que fue menor, 42,9\%. Se demuestra interés de los propietarios en mantener sus mascotas vacunadas especialmente contra la enfermedad de la rabia, pero se notó confusión en la comunidad Coyaimuna entre vacunación y desparasitación, asintiendo que ambos tienen la misma finalidad.

Finalmente es importante resaltar el riesgo que corren los propietarios y las personas que conviven entorno a los caninos que presentaron algún tipo de infección por agentes zoonóticos, debido a que pueden generarse afecciones al compartirse el mismo ambiente (27). Cuadros como Larva Migrans Visceral pudiendo ocasionar patologías como eosinofilia pulmonar, síndrome de Löeffler, dermatitis cutánea, granulomas de cuerpo extraño y ocular como diplopía e incluso ceguera por daño en retina (Uncinarias, T. canis) y cuadros diarreicos recurrentes, malnutrición, avitaminosis por Giardia spp., que son las patologías más frecuentes por agentes parasitarios zoonóticos como los hallados en el presente estudio y donde la población infantil es la de mayor riesgo de contraerlas, de igual manera personas como los adultos mayores $e$ incluso aquellas que presenten condición de inmunocompromiso, por el carácter de oportunistas que pueden tener algunos de estos o por ser considerados emergentes (7).

Estas zoonósis se pueden prevenir con cambios adecuados en el comportamiento y disminución de conductas de riesgo como lo son: la adecuada eliminación de las excretas de las mascotas, recogiéndolas de los lugares públicos en donde la mayoría de los núcleos familiares realizan sus actividades lúdicas entre semana o los fines de ésta, realizando una adecuada cocción y limpieza de los alimentos y agua de consumo diario, sumo cuidado de las mascotas lo que incluye tener completos sus tratamientos antiparasitarios y esquemas de vacunación, la esterilización en los animales es muy importante porque así se controla el incremento de la población canina, por ende disminuye el riesgo de infección, en el control de estas parasitosis es preponderante, la sensibilización en la comunidad acerca de la importancia que tiene esta problemática en la salud pública humana, mediante programas de educación que generen información necesaria y adecuada para adquirir 
buenos hábitos higiénicos y de convivencia y así disminuir el riesgo de infección por parásitos intestinales; sin embargo se siguen reportando estudios en los cuales las prevalencias de parásitos intestinales en caninos no disminuyen, como por ejemplo en caninos domiciliados del municipio de la Mesa-Cundinamarca - Colombia (28); o en otros países como Perú para la ciudad de Lima y México para las ciudades de Puerto Escondido y Oaxaca, 2014; reportando valores porcentuales que oscilan entre el $25 \%$ y el $49.44 \%$ respectivamente (29).

Esto ratifica que el parasitismo gastrointestinal canino, es un problema de salud pública en los países latinoamericanos y genera riesgo para la salud humana y que los mecanismos de seguimiento, control y prevención, son inexistentes o deficientes y las autoridades del sector salud no dan mayor importancia a esta problemática.

\section{Agradecimientos}

A la población de Coyaima, Tolima y a aquellos que hicieron posible esta investigación.

\section{Declaración de conflicto de intereses}

Los autores declaramos ausencia de conflicto de intereses en el desarrollo de esta investigación o en la publicación de este manuscrito.

\section{Fuente de financiación}

Grupo de Investigación de Parasitología y Microbiología Tropical, GIPAMT de la Universidad INCCA de Colombia.

\section{Referencias}

1. Botero J, Zuluaga N. Nemátodos intestinales de importancia médica en Colombia: ¿Un problema resuelto? Iatreia. 2001;14:47-56.Disponible en: http://www.scielo.org.co/ scielo.pid=S0120-87052011000100003\&script=sci_arttext

2. Bracciaforte R, Diaz M, Vottero V, Burstein V, Varengo H y Orsilles M. Enteroparasites in children and adolescents of a perubian community in the province of Cordoba. Acta bioquim. Clin. Latinoam. 2010;44:353-58. Disponible en: http://www.scielo.org.ar/scielo.php?script=sci_arttext\&pid $=$ S0325-29572010000300007

3. Galvan M, Rivera N, Loeza M, Avila X, Acero J, Troyo R, Bernal R. Nitazoxznide in the treatment of Ascaris lumbricuoides in rural zone of Colima, México. J. Helminthol. 2007;81:255-59. Disponible en: http://www.ncbi. nlm.nih.gov/pubmed/?term=Nitazoxznide + in+the+treatment + of + Ascaris + lumbricuoides + in + rural + zone + of + Colima $\% 2 C+$ Mexico

4. Botero D, Restrepo M. Parasitosis humanas. 5ta edición. Medellín: Corporación para investigaciones biológicas. 2012;p.93.

5. Oliveira-Sequeira T, Amarante A, Ferrari T, Nunes L. Prevalence of intestinal parasites in dogs from Sao Paulo State, Brasil. Vet Parasitol. 2002; 103:19-27. doi:10.1016/S0304-
4017(01)00575-1

6. Giraldo M, García N, Castaño J. Prevalencia de helmintos intestinales en caninos del departamento del Quindío. Biomédica. 2005;25:346-352. Disponible en: http://www.scielo.org.co

7. Llanos MM, Condori M, Ibáñez T, Loza.-Murguía MG. Parasitosis entérica en caninos (Canis familiaris) en el área urbana de Coroico, Nor yungas Departamento de La Paz, Bolivia. Journal of The Selva Andina Research Society. 2010;1(1):38-49. Disponible en: http://dialnet.unirioja.es/ servlet/articulo codigo $=4005914$

8. Fabijan $\mathrm{C}$. Indicadores de riesgo demográfico de transmisión de helmintiasis caninas en plazas de la ciudad de Buenos Aires. 1997. Disponible en: http/www.geocites.com/ Athens/Atlantis/4003/textos/parasito

9. Abril A, Penagos J. Determinación de parásitos gastrointestinales potencialmente zoonóticos en caninos de cinco municipios del departamento del Huila y riesgo para la salud pública. Trabajo de grado médico veterinario. Universidad de la Salle. 2004. Disponible en: http://es.scribd.com/doc/71023644/Parasitos-cos-enCaninos

10. Beltrán M, Tello R, Naquira C. Manual de procedimientos de laboratorio para el diagnóstico de los parásitos intestinales del hombre. Serie de normas técnicas Instituto Nacional de Salud. 2003;37:70-78. Disponible en: http://es.slideshare.net/ scorpio161/manual-de-procedimientos-de-laboratorio-parael-diagnstico-de-los-parsitos-intestinales-del-hombre

11. Fontanarosa M, Vezzani D, Basabe J, Eiras D. An epidemiological study of gastrointestinal parasites in dogs from Southern Greater Buenos Aires (Argentina): Age, Gender, breed, mixed infections and seasonal and spatial patterns. Vet Parasitol. 2010;136:283-95. Disponible en: http://www.sciencedirect.com/science/article/pii/S030440 1705005534

12. López J, Abarca K, Paredes P, Insua E. Parásitos intestinales en caninos y felinos con cuadros digestivos en Santiago de Chile. Consideraciones en salud pública. Rev. Med. Chile. 2006;134:193-200. http://dx.doi.org/10.4067/S003498872006000200009

13. Loza V, González J, Marín G. Estudio epidemiológico de Toxocara sp y Ancylostoma sp en canes de los distritos I al IV de Santacruz de la Sierra. REDVET. 2006;7:1560-71. Disponible en: http://www.veterinaria.org/revistas/ redvet/n090906/090625.pdf

14. Cabrera P, Ordoñez O, Cotes J, Rodríguez J, Villamil L. Determinación de parásitos zoonóticos (helmintos y protozoarios) en caninos del centro de zoonósis en Bogotá D.C. Biomédica. 2003;23:153.

15. Penagos J, Ardila A, Fernández J, Vargas J, Lozano C, López C. Parásitos gastrointestinales en caninos de 5 municipios del Huila y su importancia en salud pública. Infectio. 2004;8:138. Disponible en: http://es.scribd.com/doc/71023644/ Parasitos-cos-en-Caninos

16. Vásquez L, Campo V, Vergara D, Rivera O, Cordero H, Dueñas J. Prevalencia de Toxocara canis y otros parásitos intestinales en caninos de la ciudad de Popayán. Universidad del Cauca. 2004. Disponible en: http://www.engormix.com/ MA-mascotas/ sanidad/articulos/prevalencia-toxocara-canisotros-t5466/165-p0.htm

17. Solarte L, Castañeda R, Pulido A. Parásitos gastrointestinales en perros callejeros del centro de zoonósis de Bogotá D.C., Colombia. Neotrop. Helminthol. 2013.7:1. Disponible en: http://revistas.concytec.gob.pe/pdf/nh/v7n1/a09v7n1.pdf Acha P, Szyfres B. Zoonósis y enfermedades trasmisibles comunes al hombre y los animales. 3ra edición. Washington. OPS. 2003;p.398. Disponible en: http://www.paho.org/ hq/index 
19. Tortolero J, Cazorla J, Morales P, Acosta M. Prevalencia de enteroparasitos en perros domiciliarios de la ciudad de la vela, Estado Falcón. Venezuela. Revista Científica FCV-LUZ. 2005;18:312-18. Disponible en: http://www.redalyc.org/ articulo. oa?id=95918312

20. Mena L, Duarte E, Vargas J, García M, Medina R. Prevalencia de parásitos gastroentericos en la ciudad de Escárcega, Campeche. México. 2007;27(2):209-217. Disponible en: http://www.universidadyciencia.ujat.mx/sistema/documento s/volumenes/27-2-2011/560.pdf

21. Andresiuk V, Sardella N, Denegri G. Seasonal fluctuations in prevalence of dog intestinal parasites in public squares of, Mar del Plata city, Argentina and its risk for humans. Rev. Argen. Microbiol. 2007;39:221-24. Disponible en: http://www.scielo.org.ar/scielo.php?pid=S0325-75412007 000400007\&script=sci_arttext

22. Yungas N, Mariana L, Marcelina C, Teddy I, Manuel L, Articulo D. Departamento de La Paz, Bolivia Enteric Parasitic in canines ( Canis familiaris ) in the urban area of Coroico, Nor Yungas department of La Paz Bolivia Resumen Introducción Una de particularidad que se observa en la ciudad de Coroico es la vinculada al. J Selva Andin Res Soc. 2010;1(1):37-49Zarate D, Chávez A, Casas E, Falcón N. Prevalencia de Giardia sp. en canes de los distritos del cono sur de Lima Metropolitana. Rev Inv. Vet. 2008;14:134-139. http://dx.doi.org/10.15381/rivep.v14i2.1617.

23. Claerebout E, Casaert S, Dalemans A, Levecke B, Vercruysse $\mathrm{J}$, Geurden T. Giardia and other parasites in different dog's populations in northern Belgium. Vet. Parasitol. 2007: 161: 41-46. http://dx.doi.org/10.1016/j.vetpar.2008.11.024

24. Guzmán A, Jaramillo A, Loaiza J. Prevalencia de parásitos intestinales en caninos atendidos en el centro de veterinaria y zootecnia de la universidad de CES (Medellín). Revista CES/ Medicina Veterinaria y Zootecnia. 2007;2:24-31. Disponible en: http://revistas.ces.edu.co/index.php/mvz/article/ viewFile/375/1877

25. Balassiano B, Campos M, Pereira M. Factors associated with gastrointestinal parasite infection in dogs in Rio de Janeiro, Brazil. Preventy Veterinary Medicine. 2009;91:234-240. Disponible en: http://www.sciencedirect.com/science/ article/pii/S0167587709001652

26. González D, Moreno L, Hermosilla C. Parásitos en perros de San Juan Bautista, Isla Robinson Crusoe, Chile. Arch. Med. Vet.2008;40:193-195. Disponible en: http:// www.ramedveterinaria.equipu.cl/index.php/ramedveterinari a/article/viewFile/13/24.

27. Alarcón Z, Juyo V, Larrotta J. Caracterización epidemiológica de parásitos gastrointestinales zoonóticos en caninos con dueño del área urbana del municipio de la Mesa, Cundinamarca. Revista FMVZ-UN. 2015;62:1. Disponible en: http://dx.doi.org/10.15446/rfmvz.v62n1.49382

28. Serrano E, Tantalean M, Castro V, Quispe M, Casas G. Estudio retrospectivo de frecuencia de parásitos en muestras fecales en análisis rutinarios de laboratorio. Rev.Investig.vet.Peru. 2014. 25:1. Disponible en: http://www.scielo.org.pe/ scielo.php?script=sci_arttext\&pid=s1609-1172014000 100014\&lng=pt\&nrm=iso\&tlng=pt

29. Hernández L, Ganad M, Reyes K, Rojas D, Calderón M, Cruz J, Arcos J. Riesgo potencial de parásitos zoonóticos presentes en heces caninas en Puerto Escondido, Oaxaca. Rev Salud Pública de México. 2014;56:6. Disponible en: http://www.scielos.org/pdf/spm/v56n6/v56n6a12.pdf. 